\title{
BMJ Open Efficacy and safety of treatments for REM sleep behaviour disorder in Parkinson's disease: a systematic review and Bayesian network meta- analysis protocol
}

\author{
Fabin Lin (D) , ${ }^{1,2}$ Yanhong Weng, ${ }^{1,2}$ Xiaofeng Lin, ${ }^{3}$ Dihang $\mathrm{Wu},{ }^{3}$ Yixiao $\mathrm{Su},{ }^{2}$ \\ Guoen $\mathrm{Cai}^{1}$
}

To cite: Lin F, Weng $Y$, Lin $X$, et al. Efficacy and safety of treatments for REM sleep behaviour disorder in Parkinson's disease: a systematic review and Bayesian network metaanalysis protocol. BMJ Open 2021;11:e047934. doi:10.1136/ bmjopen-2020-047934

- Prepublication history and additional supplemental material for this paper are available online. To view these files, please visit the journal online (http://dx.doi.org/10.1136/ bmjopen-2020-047934).

Received 12 December 2020 Accepted 09 November 2021

A) Check for updates

(c) Author(s) (or their employer(s)) 2021. Re-use permitted under CC BY-NC. No commercial re-use. See rights and permissions. Published by BMJ.

${ }^{1}$ Department of Neurology, Fujian Medical University Union Hospital, Fuzhou, Fujian, People's Republic of China ${ }^{2}$ Fujian Medical University, Fuzhou, Fujian, People's Republic of China

${ }^{3}$ Provincial Medical College, Fujian Medical University, Fuzhou, Fujian, People's Republic of China

Correspondence to

Dr Guoen Cai;

cgessmu@fjmu.edu.cn

\section{ABSTRACT}

Introduction Sleep disorders are the main non-motor characteristics of Parkinson's disease (PD). The quality of life is significantly impacted by rapid eye movement sleep behaviour disorder (RBD). It is not clearly evidenced in the literature that some medications can reduce the dream activities of patients with PD and RBD and improve sleep quality. And, they have side effects that may increase the severity of this disease. To further understand which medication has better efficacy and fewer adverse effects for patients with $\mathrm{PD}$ and $\mathrm{RBD}$, it is necessary to perform a network meta-analysis.

Methods and analysis This protocol is performed accordingly to the guidelines of the Preferred Reporting Items for Systematic Review and Meta-Analysis Protocols and the Cochrane Collaboration Handbook.

A thorough literature selection will be conducted up to September 2021 using PubMed, Cochrane Library (The Cochrane Database of Systematic Reviews) and Embase. We will not only include randomised controlled trials, but prospective, retrospective cohort, case-control, nested case-control, case-cohort, cross-sectional and case series. We will use the Cochrane Collaboration tool to assess the risk of bias. Pairwise and network metaanalyses will be conducted using the $\mathrm{R}$ netmeta package and Stata V.14.0. The relative ranking probability of the best intervention will be estimated using the surface under the cumulative ranking curve. Additionally, sensitivity analysis, subgroup analysis, quality assessment and publication bias analysis will be performed.

Ethics and dissemination No research ethics approval is required for this systematic review, as no confidential patient data will be used. We will disseminate our findings through publication in a peer-reviewed journal and conference presentations, and our review will support development of a BMJ Rapid Recommendations providing contextualised clinical guidance based on this body of evidence.

PROSPERO registration number CRD42020206958.

\section{INTRODUCTION}

Rapid eye movement sleep behaviour disorder (RBD), which is associated with Parkinson's
Strengths and limitations of this study

- This study will be the first network meta-analysis comparing the efficacy and the safety of multiple regimens for treating patients with rapid eye movement and Parkinson's disease.

- Both pairwise and network meta-analyses will be performed.

- Our results will be limited by the number of available trials and the quality of the included trials.

disease (PD) and other synucleinopathies, is a common non-motor manifestation. ${ }^{1}$ $\mathrm{RBD}$ is a precursor to some neurological diseases, and $70 \%$ of patients with RBD may develop PD. ${ }^{23} \mathrm{RBD}$ is a rapid eye movement (REM) sleep-related parasomnia and also a type of dream-related complex motor activities. ${ }^{4}$ Patients with RBD exhibit dream-like behaviours. The dreams are usually accompanied by unpleasant and violent content. RBDinduced motor activities-such as twitching limbs ranging from simple to more complex, more aggressive and violent twitching-manifest as intense sleep talk, punching, kicking, sleepwalking and other violent behaviours; these sports behaviours may cause patients to inflict harm to themselves or their sleeping partners. $^{56}$

A consensus on the management of RBD was released in 2013, in which a variety of medications was proposed, including melatonin, clonazepam, ramelteon, pramipexole and so on. Melatonin may improve sleep efficiency, and the antioxidant function of melatonin has a protective effect on nerves. ${ }^{78}$ Melatonin may be used to treat RBD by regulating gamma-aminobutyric acid inhibition and stabilising circadian rhythms. ${ }^{9}$ Clonazepam may reduce the muscle twitches of RBD, 
demonstrating a positive effect on idiopathic RBD, ${ }^{10}$ but its side effects are also obvious-such as daytime sleepiness, cognitive dysfunction in the elderly and worsening of sleep apnoea. Those symptoms are dangerous side effects for patients with PD. Ramelteon is a selective activation of melatonin type 1 and type 2 receptors. ${ }^{11}$ The pathology of RBD and PD has a common mechanism of dopamine loss. ${ }^{12}{ }^{13}$ Sometimes the effect of dopamine receptor agonists is significant in RBD. For example, pramipexole may improve RBD symptoms, which may be related to changing the content of dreams or reducing REM density. ${ }^{14}$ But most of its therapeutic effects are still unclear. Study has shown that it promotes sleep efficiency at low doses, whereas at high doses, it may cause sleep disturbances, aggravate the condition and greatly reduce the efficacy of the medication. ${ }^{15}$ Several review articles have already been published on formal compilation, summary or evaluation to assess the effectiveness and side effect of the medications. ${ }^{16-18}$ But, this lack of meta in the field on whether the commonly prescribed medications are indeed efficacious and safe for treating RBD.

Frequentist method interprets probability as a statistical mean (law of large numbers) through a large number of independent experiments; Bayesian method interprets probability as a degree of belief (no need for a large number of experiments). The interpretation of the Bayesian method is very useful when the number of trials considered is small.

To provide individualised medication treatment options for patients with PD associated with REM sleep disorder, we summarised the effectiveness and the safety of multiple medications using a systematic review and a Bayesian network meta-analysis of existing literature.

\section{OBJECTIVE}

This systematic review and network meta-analysis aimed to estimate the comparative clinical efficacy and safety of treatments for REM sleep behaviour disorder in PD.

\section{METHODS}

This network meta-analysis has been prospectively registered in the PROSPERO database (available at http:// www.crd.york.ac.uk/PROSPERO/display_record.php? $\mathrm{ID}=\mathrm{CRD} 42020206958$ ). The protocol is prepared using the Preferred Reporting Items for Systematic Review and Meta-Analysis Protocols (see online supplemental table 1). ${ }^{192}$

\section{Type of study}

All of the included studies will contain comparisons between the therapeutic effects of one active medication and another or placebo. Studies for which data are unavailable will be eliminated. We will include randomised controlled trials, prospective, retrospective cohort, case-control, nested case-control, case-cohort, cross-sectional and case series. Comments, editorials,

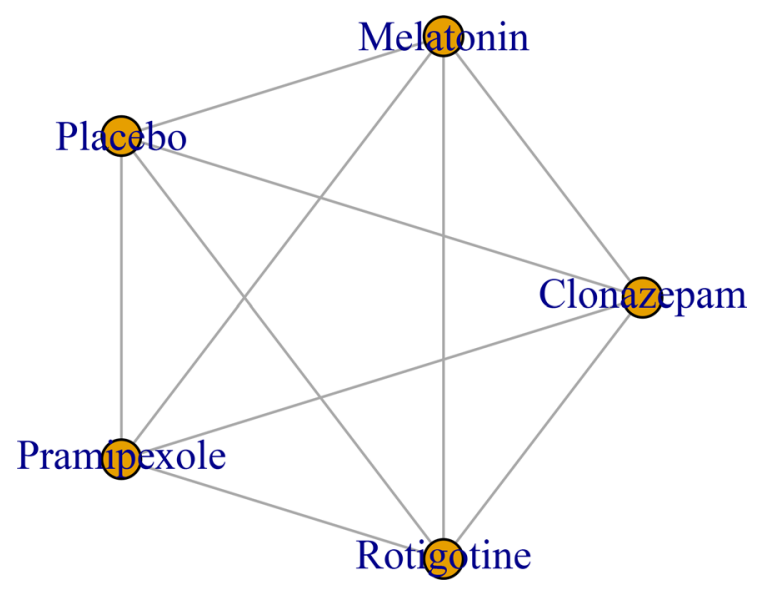

Figure 1 An ideal network plot for the outcome.

letters to the editor, conference abstract and animal studies will be excluded.

\section{Type of participant}

Participants will include men and non-pregnant/nonbreastfeeding women $\geq 18$ years of age at the time of screening; had a diagnosis of idiopathic PD (modified Hoehn and Yahr (H\&Y) criteria stages 2-4); and could observe RBD symptoms and provide information to investigators.

\section{Type of intervention}

In our study, four medications and placebo will be compared, including rotigotine, pramipexole, clonazepam and melatonin. We will access information on relevant interventions from placebo-controlled and head-to-head trials. An ideal network plot, which is a fully connected network with all of the expected interventions, was generated (figure 1).

\section{Type of outcomes}

Trials that contained at least one of the following outcomes will be included:

\section{Primary outcomes}

- The change from baseline in the Clinical Global Impression-Improvement score.

\section{Secondary outcomes}

- The change from baseline in the Clinical Global Impression-Severity.

- The change from baseline in the Korean Epworth Sleepiness Scale.

- The change from baseline in the Parkinson's Disease Sleep Scale.

- The change from baseline in the Korean Version of the Montreal Cognitive Assessment.

- The change from baseline in the Unified Parkinson's Disease Rating Scale.

This systematic review and network meta-analysis will be based on published trials, and patients and the public are not involved in this protocol. 


\begin{tabular}{|c|c|}
\hline$\# 1$ & $\begin{array}{l}((((((C l o n a z e p a m) \text { OR }(2 \mathrm{H}-1,4-B e n z o d i a z e p i n-2-o n e, 5-(2-c h l o r o p h e n y l)-1,3-d i h y d r o-7-n i t r o-)) \\
\text { (Klonopin)) OR (Antelepsin)) OR (Ro 5-4023)) OR (Ro 54023) }\end{array}$ \\
\hline \#2 & $\begin{array}{l}(((((((((\text { Pramipexole) OR (4,5,6,7-Tetrahydro-N6-propyl-2,6-benzothiazole-diamine)) OR (Pramipexol)) OR (2-Amino- } \\
4,5,6,7-\text { tetrahydro-6-propylaminobenzothiazole)) OR (Dexpramipexole)) OR (Pramipexol, (R)-isomer)) OR (Mirapex)) } \\
\text { OR (Pramipexol Dihydrobromide, ( }( \pm) \text {-isomer)) OR (Pramipexol Dihydrobromide, (S)-isomer)) OR (Pramipexole } \\
\text { Dihydrochloride Anhydrous) }\end{array}$ \\
\hline \#3 & (Melatonin) \\
\hline$\# 4$ & $((((($ Rotigotine) OR (rotigotine, $( \pm)-))$ OR (racemic N-0437)) OR (rotigotine $( \pm)$-form)) OR (Rotigotine CDS)) OR (Neupro) \\
\hline$\# 5$ & $\begin{array}{l}\text { (((Idiopathic Parkinson's Disease) OR Lewy Body Parkinson Disease) OR Lewy Body Parkinson’s Disease) OR } \\
\text { Primary Parkinsonism) OR Parkinsonism, Primary) OR Parkinson Disease, Idiopathic) OR Parkinson's Disease) } \\
\text { OR Parkinson's Disease, Idiopathic) OR Parkinson's Disease, Lewy Body) OR Idiopathic Parkinson Disease) OR } \\
\text { Paralysis Agitans)) OR “Parkinson Disease)) }\end{array}$ \\
\hline \#6 & $\begin{array}{l}((((((\text { REM Sleep Behavior Disorder) OR (Behavior Disorder, REM)) OR (Behavior Disorders, REM)) OR (REM Behavior } \\
\text { Disorders)) OR (REM Behavior Disorder)) OR (Behavior Disorder, Rapid Eye Movement Sleep)) OR (Rapid Eye } \\
\text { Movement Sleep Behavior Disorder) }\end{array}$ \\
\hline \#7 & \#1 OR \#2 OR \#3 OR \#4 \\
\hline \#8 & \#5 AND \#6 \\
\hline \#9 & \#7 AND \#8 \\
\hline
\end{tabular}

\section{Study selection and search strategy}

We will search three databases, including PubMed, Cochrane Library (The Cochrane Database of Systematic Review) and Embase, and the search will be limited from their inception to September 2021, with no restriction on publication states or language. Studies that are included in published systematic reviews and meta-analyses will also be added to this study if they are not obtained by other methods. The search strategy is described in table 1 .

\section{Study selection}

The titles and abstracts of the searched studies will be imported into EndNote V.X5.0.1, then screened and selected independently by two reviewers. Trials will be excluded if both investigators determine that the study does not meet the inclusion criteria. We will also obtain full texts if the trials cannot be identified by titles and abstracts. Any discrepancies will be discussed among two reviewers, and the trial will be included if two of the reviewers reach consensus.

\section{Data extraction}

The data of the included studies will be extracted into a predetermined sheet and entered into Microsoft Excel V.2019. Two investigators will extract the following information according to the predetermined table:

- Study design (authors, countries, publication date, follow-up durations and number of participants);

- Patient characteristics (age, sex, baseline H\&Y, baseline Clinical Global Impressions- Improvement (CGII)); and

- Interventions (medications and dosages).

\section{Risk of bias of individual studies}

The risk of bias of the included studies will be assessed using the Cochrane risk of bias tool for randomised controlled trials and observational studies. ${ }^{21}$ Six fields will be assessed: random sequence generation, allocation concealment, blinding of participants and personnel, blinding of outcome assessment, incomplete outcome data and selective reporting. Three different evaluations will be performed: high risk, low risk and unclear risk. Two reviewers will independently conduct quality assessment, and any disagreement will be resolved by discussion with another author.

\section{STATISTICAL ANALYSIS}

\section{Pairwise meta-analysis}

We will use Stata V.14.0 (StataCorp) to perform a traditional pairwise meta-analysis. We will use a random effects or fixed effects model to calculate the OR and the pooled estimate of the $95 \%$ CI of the direct comparison between the two strategies. If heterogeneity does not exist, we will use a fixed effects model to summarise the results in the study; otherwise, we will use a random effects model.

\section{Bayesian network meta-analysis}

To integrate direct and indirect comparisons, we will conduct the network meta-analysis within a Bayesian framework using Markov chain Monte Carlo methods in the R-netmeta package, using four chains with overdispersed initial values and Gibbs sampling based on 50 000 iterations after a burn-in phase of 10000 iterations. ${ }^{22}$ Continuous outcomes will be calculated and expressed as the mean difference, and the effect measure for dichotomous outcomes will be used as the $\log$ OR. Both will be calculated with their associated 95\% CIs. Otherwise, a predictive probability of the best intervention will be estimated using the surface under the cumulative ranking curve. 


\section{Dealing with missing data}

To obtain missing data, we will first attempt to contact the authors by email. Otherwise, the data will be verified from other trials in the network or from other published meta-analyses.

\section{Measures for transitivity assumption}

Clinical and methodological similarities are most commonly used to assess transitivity between eligible trials. Similarities in clinical factors mainly will include baseline characteristics of patients (ie, population, intervention and comparison characteristics), follow-up time and clinical outcomes. The design and quality of each eligible study are methodological similarities. We will assess the transitivity of the included studies according to the above factors.

\section{Investigation of heterogeneity}

$\mathrm{I}^{2}$ statistics will be used to assess heterogeneity among studies. We will consider a value of $\mathrm{I}^{2}$ from $0 \%$ to $24 \%$, $25 \%$ to $50 \%$ and greater than $50 \%$ as low, moderate and high heterogeneity, respectively. A meta-regression model will be used to explore reasons for any high heterogeneity.

\section{Measures for inconsistency}

To compare the consistency and inconsistency models, we will use the deviance information criterion (DIC). The DIC provides a measure of model fit that penalises model complexity; lower values of DIC indicate a better fit of the model, and the difference value of two models is material. In addition, the $p$ value of the node-split analysis, which is derived from a comparison between the direct estimate value and the indirect estimate value, can be used to evaluate the consistency of the network. A $p<0.05$ will be used to indicate significant inconsistencies. ${ }^{23}$

\section{Measures for publication bias}

The small study effect for the entire network will be assessed by constructing a comparison-adjusted funnel plot considering different comparisons. In the absence of a small study effect, the studies will form an inverted funnel centred at 0 .

\section{Subgroup analyses and sensitivity analyses}

To assess the robustness of the findings of our primary efficacy outcome, we will perform multiple sensitivity analyses. ${ }^{24}$ These will include the following:

- Exclusion of studies that were published before 2000;

- Exclusion of studies that have small-study effects;

- Exclusion of studies that have attrition bias; and

- Exclusion of studies that have reporting bias.

Additionally, we will perform a meta-regression analysis, which will include the following:

- Publication date of the studies;

- Sex, age, disease duration, baseline CGI-I scores and baseline H\&Y scores of the patients; and

- Trial duration.

\section{ETHICS AND DISSEMINATION}

No ethical concerns. The findings will be disseminated through a designated website, publications, presentations in webinars and social media.

Contributors GC is responsible for this research. GC and FL conceived and designed this study. GC, FL, YW, DW, YS and XL participated in drafting the agreement and preparing the manuscript. GC, FL, YW, DW, YS and XL read and approved the final manuscript.

Funding This meta-analysis was supported by internal funds and did not involve any business affairs.

Competing interests None declared.

Patient consent for publication Not applicable.

Provenance and peer review Not commissioned; externally peer reviewed.

Supplemental material This content has been supplied by the author(s). It has not been vetted by BMJ Publishing Group Limited (BMJ) and may not have been peer-reviewed. Any opinions or recommendations discussed are solely those of the author(s) and are not endorsed by BMJ. BMJ disclaims all liability and responsibility arising from any reliance placed on the content. Where the content includes any translated material, BMJ does not warrant the accuracy and reliability of the translations (including but not limited to local regulations, clinical guidelines, terminology, drug names and drug dosages), and is not responsible for any error and/or omissions arising from translation and adaptation or otherwise.

Open access This is an open access article distributed in accordance with the Creative Commons Attribution Non Commercial (CC BY-NC 4.0) license, which permits others to distribute, remix, adapt, build upon this work non-commercially, and license their derivative works on different terms, provided the original work is properly cited, appropriate credit is given, any changes made indicated, and the use is non-commercial. See: http://creativecommons.org/licenses/by-nc/4.0/.

ORCID iD

Fabin Lin http://orcid.org/0000-0001-7628-9152

\section{REFERENCES}

1 Boeve BF, Silber MH, Ferman TJ, et al. Association of REM sleep behavior disorder and neurodegenerative disease may reflect an underlying synucleinopathy. Mov Disord 2001;16:622-30.

2 Iranzo A, Molinuevo JL, Santamaría J, et al. Rapid-eye-movement sleep behaviour disorder as an early marker for a neurodegenerative disorder: a descriptive study. Lancet Neurol 2006;5:572-7.

3 Gagnon J-F, Postuma RB, Mazza S, et al. Rapid-eye-movement sleep behaviour disorder and neurodegenerative diseases. Lancet Neurol 2006;5:424-32.

4 Porter VR, Avidan AY. Clinical overview of REM sleep behavior disorder. Semin Neurol 2017;37:461-70.

5 Schenck CH, Hurwitz TD, Mahowald MW. Symposium: normal and abnormal REM sleep regulation: REM sleep behaviour disorder: an update on a series of 96 patients and a review of the world literature. J Sleep Res 1993;2:224-31.

6 Olson EJ, Boeve BF, Silber MH. Rapid eye movement sleep behaviour disorder: demographic, clinical and laboratory findings in 93 cases. Brain 2000;123:331-9.

7 McGrane IR, Leung JG, St Louis EK, et al. Melatonin therapy for REM sleep behavior disorder: a critical review of evidence. Sleep Med 2015;16:19-26.

8 St Louis EK, Boeve AR, Boeve BF. Rem sleep behavior disorder in Parkinson's disease and other synucleinopathies. Mov Disord 2017;32:645-58.

9 McCarter SJ, Boswell CL, St Louis EK, et al. Treatment outcomes in REM sleep behavior disorder. Sleep Med 2013;14:237-42.

10 Li SX, Lam SP, Zhang J, et al. A prospective, naturalistic followup study of treatment outcomes with clonazepam in rapid eye movement sleep behavior disorder. Sleep Med 2016;21:114-20.

11 Kato K, Hirai K, Nishiyama K, et al. Neurochemical properties of ramelteon (TAK-375), a selective MT1/MT2 receptor agonist. Neuropharmacology 2005;48:301-10.

12 Iranzo A, Lomeña F, Stockner $\mathrm{H}$, et al. Decreased striatal dopamine transporter uptake and substantia nigra hyperechogenicity as risk markers of synucleinopathy in patients with idiopathic rapid-eyemovement sleep behaviour disorder: a prospective study [corrected]. Lancet Neurol 2010;9:1070-7. 
13 Albin RL, Koeppe RA, Chervin RD, et al. Decreased striatal dopaminergic innervation in REM sleep behavior disorder. Neurology 2000;55:1410-2.

14 Sasai T, Inoue Y, Matsuura M. Effectiveness of pramipexole, a dopamine agonist, on rapid eye movement sleep behavior disorder. Tohoku J Exp Med 2012;226:177-81.

15 Tan SM, Wan YM. Pramipexole in the treatment of REM sleep behaviour disorder: a critical review. Psychiatry Res 2016;243:365-72.

16 Gilat M, Marshall NS, Testelmans D, et al. A critical review of the pharmacological treatment of REM sleep behavior disorder in adults: time for more and larger randomized placebo-controlled trials. $J$ Neurol 2021. doi:10.1007/s00415-020-10353-0. [Epub ahead of print: 07 Jan 2021].

17 Videnovic A, Ju Y-ES, Arnulf I, et al. Clinical trials in REM sleep behavioural disorder: challenges and opportunities. J Neurol Neurosurg Psychiatry 2020;91:740-9.

18 Aurora RN, Zak RS, Maganti RK, et al. Best practice guide for the treatment of REM sleep behavior disorder (RBD). J Clin Sleep Med 2010;6:85-95.
19 Moher D, Liberati A, Tetzlaff J, et al. Preferred reporting items for systematic reviews and meta-analyses: the PRISMA statement. PLoS Med 2009;6:e1000097

20 Shamseer L, Moher D, Clarke M, et al. Preferred reporting items for systematic review and meta-analysis protocols (PRISMA-P) 2015: elaboration and explanation. BMJ 2015;349:10.1136/bmj. g7647:g7647.

21 Higgins JPT, Altman DG, Gøtzsche PC, et al. The Cochrane collaboration's tool for assessing risk of bias in randomised trials. BMJ 2011;343:d5928.

22 Jansen JP, Crawford B, Bergman G, et al. Bayesian meta-analysis of multiple treatment comparisons: an introduction to mixed treatment comparisons. Value Health 2008;11:10.1111/j.1524-4733.2008.00347.x:956-64.

23 Dias S, Welton NJ, Caldwell DM, et al. Checking consistency in mixed treatment comparison meta-analysis. Stat Med 2010;29:932-44.

24 Higgins JPT, Thompson SG, Deeks JJ, et al. Measuring inconsistency in meta-analyses. BMJ 2003;327:10.1136/ bmj.327.7414.557:557-60. 